\title{
Evaluación direccional del cono de humedecimiento en suelos ferralíticos rojos y francos utilizando sistemas de riego subsuperfical en la caña de azúcar
}

\author{
Nestor Mendez Jurjo \\ Albi Mujica Cervantes, Dayamira Mundiel Sotolongo \\ nestorm@unica.cu \\ Universidad de Ciego de Ávila (Universidad $\overline{\text { Certificada) Cuba }}$
}

Recibido: septiembre 2016. Aprobado: diciembre 2016

\section{RESUMEN}

Los Valores alcanzados por el Coeficiente de Variación (CV), se encuentran comprendidos entre el $1.2 \%$ y el 2.6\% comprobándose que la calidad del emisor es buena. Las máximas variaciones del caudal del emisor ocurrieron cuando la carga alcanzó valores superiores a los 20 mca, las menores variaciones se presentaron cuando la carga resulto de 5 mca, evidenciando el nivel de compensación del emisor estudiado. Se estudió, además, las características hidroedafológica donde el medio (manguera) deja pasar el agua a través de ella por unidad de área transversal a la dirección del flujo en suelos ferralíticos rojos y textura moderadamente gruesos de diferentes tipos de suelos con fines de diseños para estos sistemas, determinándose las formas y dimensiones del bulbo húmedo, obteniéndose que la longitud del diámetro de humedecimiento se estabiliza a partir de los 5.2 litros con $48 \mathrm{~cm}$ en suelos ferralíticos rojos, el espaciamiento entre emisores pueden garantizar una franja continua de humedad y traslape entre bulbos de un 10\%, para los suelos francos la longitud del diámetro de humedecimiento se estabiliza a partir de los 5.1 litros a unos $46 \mathrm{~cm}$ por lo que el espaciamiento entre emisores garantiza franjas continua de humedad y el traslape entre bulbos de un $8 \%$. Finalmente se realizó un estudio con el propósito de conocer la cantidad de agua suministrada al suelo por por el emisores, obteniéndose resultados satisfactorios dado que la tecnología aporta elevados rendimientos agrícolas, mínimos gastos de agua y energía, favoreciendo la preservación del medio ambiente y recursos naturales. Palabras claves: Coeficiente de Variación, Emisor, Humedecimiento, suelos.

\section{SUMARY}

In This work are presented the results obtained in the tecnique hidraulic evaluation of the emittter type dropfeeed model DRIPNET autocompensating made by the firm NETAFIM FROM Israel .The values reached by the variation coefficient (VC) varies between 1,2| \% verifying that the quality of the emitter can be evaluated as good. The greater variations of the flow of the emitter occured when the charge reached valves higher to 20 mca and the lesser variations were present when the charge was only of 5 mca. This aspect was evidenced by the compensation level of the emitter studied. Besides, the hidroedaphologics characteristics of the different types of soils were studied in order to design these systems, determining the forms and dimensions of the wet bulb, obtaining a stabilization of the moisture diameter at 5,2 liters at $48 \mathrm{~cm}$ in red ferralitic. so the space of the emitter can guarentee a continuoos flow of moisture between bulbs of $10 \%$.determining the forms and dimensions of the wet bulb, obtaining a stabilization of the moisture diameter at 5,1 liters at $46 \mathrm{~cm}$ sandy and muddy. so the space of the emitter can guarentee a continuous flow of moisture between bulbs of $8 \%$. Finally, a study was performed in order to know the amount of water supplied to the soil by the emitter, obtaining good results due 
that this technology gives high efficiency with minimum consumption of water and energy, preserving in this way our environment and natural resources. Key word: variation coefficient emitter, humectate, soils.

\section{INTRODUCCIÓN}

El rápido crecimiento de la población mundial ha hecho que el empleo eficiente del agua de riego sea de importancia vital, particularmente en los países más pobres donde el mayor potencial para aumentar la producción alimentaria y los ingresos rurales se encontrarían frecuentemente en las zonas de riego, (Tornería et al, 2005).

Según Aidi et al, (2007) presidente de IRRIFRANCE, el agua será el factor desarrollo duradero, cabe destacar que la distribución de recursos de agua por persona no es uniforme en todo el mundo. Es por eso que en varios países, algunos de los cuales se encuentran entre los grandes productores agrícolas, la búsqueda el buen uso y el dominio del agua resultan indispensables. El riego moderno posibilita un ahorro de aproximadamente el $55 \%$ del más determinante del consumo de agua por los métodos tradicionales.

La escasez de agua se ha convertido en una crisis a nivel mundial; siendo sus causas la presión sobre los recursos hídricos debido a la sobrepoblación, la contaminación, el cambio en los usos y ciclos del agua, y el cambio climático generado en gran parte por los seres humanos (FAO, 2008).

La tecnología de riego por goteo subsuperficial se ha utilizado con éxito en la UBPC cañera para obtener mejores respuestas en cuanto a su crecimiento, desarrollo y aumentar la producción con bajos costos, está demostrado que la caña con un buen manejo del riego aumenta considerablemente sus rendimientos, utilizando emisores de tipo integrado (goteros) (Ruiz et al, 1999).

La práctica de reposición artificial de agua al suelo o riego de las tierras es muy antiguo, la eficiencia con que se realiza varía sustancialmente entre valores tan altos como $90 \%$ y tan bajo como $25 \%$. Por otra parte, en ocasiones aún con elevada eficiencia de aplicación se tiene baja eficiencia de distribución y/o almacenamiento en el suelo, dando por resultado una irregular distribución de la humedad y como consecuencia baja mucho la producción de la planta. (Rodríguez S. F. et, al. 1982).

El déficit de agua influye en forma negativa sobre el crecimiento y los rendimientos del cultivo de la caña de azúcar (Martínez y Ortega, 1986; Young et al. 2007).

En muchas regiones del planeta el uso inapropiado del agua está amenazando la sustentabilidad de la agricultura de riego (Peña et al, 2000). Un factor que frecuentemente contribuye al mal manejo del riego es un servicio inadecuado de la distribución del agua, ocasionado por varias causas, como son 
el deterioro de la infraestructura existente, ineficiente trabajo de mantenimiento, inadecuadas políticas de manejo, inexistencia de un programa de capacitación de usuarios, esto ha contribuido a que se obtengan bajas eficiencias del uso del agua (Peña et al., 1999).

De acuerdo con Medina et al, (1997) los emisores se clasifican en: miniaspersores o difusores, goteros y tuberías de goteo. Los goteros se caracterizan por la aplicación del agua en un punto sobre o debajo de la superficie del suelo (Bliesser et al. 2000a), existiendo varios tipos de goteros (Bliesser et al, 2000b). Los goteros presentan características hidráulicas que producen pérdidas de cargas al conectar los mismos de la forma siguiente: goteros de trayectoria larga, goteros de orificio, goteros vórtice, goteros compuestos y goteros autocompensantes. Los miniaspersores se clasifican a su vez en miniaspersores fijos y miniaspersores rotatorios (Bliesser et al, 2001).

Un emisor consiste en un disipador de presión construido especialmente para generar una pérdida localizada induciendo vorticidad, fricción hidráulica o una combinación de ambas (Medina et al, 1997).

El emisor es el elemento más importante de un sistema de microirrigación, siendo su función principal permitir la salida del agua con un caudal controlado y en puntos del cultivo beneficiado previamente determinado. Un emisor consiste en un disipador de presión construido especialmente para generar una pérdida localizada induciendo vorticidad, fricción hidráulica o una combinación de ambas (Medina et al, 1997).

El objetivo es evaluar el comportamiento del Cono de Humedecimiento en los suelos ferralíticos rojos y francos utilizando emisores DRIPNET® en el riego subsuperficial bajo las condiciones edafoclimaticas típicas.

\section{MATERIAL Y MÉTODOS}

La investigación se desarrolló en la UBPC la institución perteneciente al Municipio Ciro Redondo de la Provincia de Ciego de Ávila y evaluado en la Universidad Máximo Gómez Báez Km 9.5 Provincia de Ciego de Ávila.

El sistema de microirrigación subterránea está conformado por tuberías laterales con emisores integrados, tuberías distribuidoras, tuberías secundarias y principales, sistemas de filtrado y fertirrigación y estación de bombeo, además de ello, el sistema ha sido diseñado con un nivel de 
automatización que posibilita realizar determinadas actividades de operación de forma automática, constituyendo el mismo un sistema de los llamados "sistemas inteligentes".

En la figura 1 la disposición de la tubería lateral soterrada por hilera de plantas y la ubicación del sistema radical con respecto al volumen de suelo humedecido.

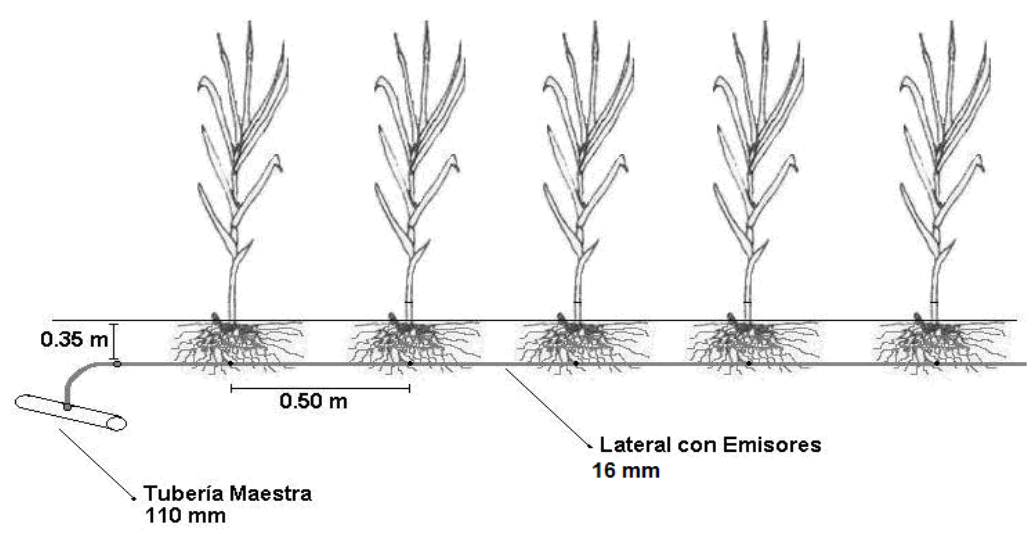

Figura 1. Disposición de la tubería lateral soterrada por hilera de planta

\section{Determinación de las características técnico-hidráulicas del emisor}

Para la determinación de las características técnico-hidráulicas del emisor tipo gotero modelo DRIPNET® autocompensantes fabricado por la firma NETAFIM de procedencia Israelí, se realizaron experimentos de laboratorio para lo cual se utilizó un tubería lateral conformada por 5 emisores integrados a la misma y espaciados a $0.50 \mathrm{~m}$ cada uno. Se realizaron mediciones del caudal entregado por los emisores a las presiones siguientes: 5, 10, 15, 20, 25 y 30 mca para lo cual se utilizó un manómetro tipo bordón y se recolectó el caudal entregado en un recipiente colocado debajo de cada uno de ellos los cuales fueron medidos posteriormente con una probeta graduada y expresado en litros, el tiempo de descargas se midió utilizando un cronómetro, obteniéndose finalmente el caudal descargado para cada presión. Se realizaron 5 repeticiones para los diferentes suelos. La temperatura se midió antes y después de cada medición. Con la información obtenida en cada experimento se determinó: el volumen promedio $\left(\mathrm{V}_{\mathrm{M}}\right)$, con la ecuación 2.1, el caudal promedio $\left(\mathrm{q}_{\mathrm{m}}\right)$ con la ecuación 2.2, la desviación estándar (S) con la ecuación 2.3; el coeficiente de variación de fabricación (CV) con la ecuación 2.4; el coeficiente de uniformidad (CU) mediante la ecuación 2.5, propuesta por (Christiansen et al, 1992) y el error estándar (Ee) aplicando la ecuación 2.6. 
$V m=\frac{\sum_{n}^{i=1} V i}{n}$

donde:

$\mathrm{Vm}=$ volumen promedio $(\mathrm{L})$.

$\mathrm{Vi}=$ volumen observado $(\mathrm{L})$

n. = número de observaciones.

$$
q m=\frac{\sum_{i=1}^{n} q i}{n}
$$

donde:

$\mathrm{q}_{\mathrm{m}}=$ caudal promedio $(\mathrm{L} / \mathrm{h})$

$\mathrm{q}_{\mathrm{i}}=$ caudal observado $(\mathrm{L} / \mathrm{h})$

$S=\sqrt{\frac{\sum_{i=1}^{n}\left(q-q_{n}\right)^{2}}{n-1}}$

donde:

$\mathrm{S}=$ Desviación Standard.

$$
C V=\frac{S}{q_{m}}
$$

donde:

$\mathrm{CV}=$ coeficiente de variación de fabricación (decimal).

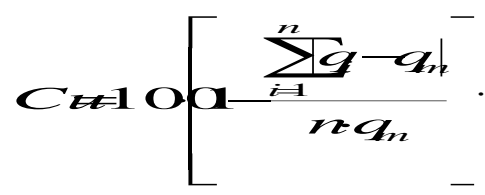

donde:

$\mathrm{Cu}=$ coeficiente de uniformidad $(\%)$.

$\mathrm{q}_{\mathrm{i}}=$ caudal de emisor $\mathrm{i}(\mathrm{L} / \mathrm{h})$.

$\mathrm{q}_{\mathrm{m}}=$ caudal promedio $(\mathrm{L} / \mathrm{h})$.

$\mathrm{n}=$ número de emisores. 


$$
E e=\frac{S}{\sqrt{n}}
$$

donde:

$\mathrm{Ee}=$ error Standard

\section{Determinación de las formas y dimensionas del bulbo húmedo del emisor dripnet con fines de diseño.}

Para la determinación de las características hidráulicas del suelo se utilizó el método de Shani et al, (1996), para lo cual, se midió los radios de humedecimiento de los emisores para cada uno de los siguientes tiempos de aplicación: 1 minutos, 5 minutos, 10 minutos, 20 minutos, 30 minutos. Los bordes del área humedecida se determinaron visualmente. Un promedio del diámetro fue estimado midiendo el diámetro observado en diferentes direcciones.

Finalizado el tiempo de aplicación se abrió una zanja paralela a la línea regante se tomaron medidas del diámetro de humedecimiento donde fue realizado un grillado con cuadriculas de áreas conocidas que permitieran medir con mayor precisión las dimensiones del bulbo de humedecimiento .Para obtener una mayor precisión en el diseño del sistema es necesario realizar ensayos de capilaridad del suelo para con ello inferir los bulbos de humedecimiento y espaciamiento de los goteros Cuando se presentó esta situación lo que se realizó fue un muestreo posterior para determinar la humedad gravimétrica en varios puntos situados según una línea horizontal, a una profundidad conveniente, donde los puntos extremos se situaron claramente fuera del bulbo para así precisar el contorno.

Los suelos donde se practicó la investigación son ferralíticos rojos (eutricos) y suelos francos con textura moderadamente fina (franco arcilloso), con $40 \%$ de arena, $35 \%$ de limo y $30 \%$ de arcilla, de acuerdo a las partículas según su tamaño.

\section{RESULTADO Y DISCUSIÓN}

\section{Radio de humedecimiento vs. Volumen de agua aplicado por el emisor modelo dripnet en suelos ferraliticos rojos eütricos y suelos francos}

En los cuadros 1 y 2, y en las figuras 3.3 y 3.4 se presentan los resultados obtenidos de la relación entre el radio de humedecimiento y el volumen de agua aplicada por el emisor DRIPNET en suelos Ferralíticos Rojos y Francos Del análisis de regresión realizado se infiere que las ecuaciones de 
más ajustes a los datos resulta una ecuación de tipo exponencial para los suelos Ferralíticos Rojos y para los suelos Francos se forman ecuaciones Lineales obtenidas en la figura 3.4

$$
R h=a \cdot V^{m}
$$

Donde:

Rh. - Radio de humedecimiento (cm).

v. - Volumen de agua aplicada (L).

a y m. - Valores obtenidos al ajustar la ecuación de regresión

Con coeficientes de regresión mayores de $94 \%$ que resultan

Valores aceptables.

Para los suelos arcillosos se pudo observar que la longitud del diámetro de humedecimiento tiende a estabilizarse a partir de los 5.2 Litros a $48 \mathrm{~cm}$, lo que quiere decir que el máximo espaciamiento entre emisores que puede garantizar una franja continua de humedad y un solapamiento entre bulbos de un $10 \%$, sería el espaciamiento entre emisores de $48 \mathrm{~cm}$.

Para los suelos Francos observamos que la longitud del diámetro de humedecimiento tiende a reducirse y se estabiliza a partir de los 5.1 Litros a $46 \mathrm{~cm}$, lo que quiere decir que el máximo espaciamiento entre emisores se puede garantizar una franja continua por lo que respecta a humedad y solapamiento entre los bulbos es de un $8 \%$.

Todo lo anterior demuestra que los suelos ferralíticos rojos son más efectivos en cuanto al área mojada horizontalmente que los suelos Francos, pero se demuestra en ambos casos el nivel de traslape que ocurre en estos suelos teniendo en cuenta la descarga de los emisores y la textura de los mismos.

Cuadro 1: Radio de humedecimiento y volumen en Suelos Ferralíticos Rojos.

\begin{tabular}{|c|c|c|c|c|}
\hline \multicolumn{5}{|c|}{} \\
\hline Emisor & Presión & Tiempo & Volumen (L) & Radio (cm) \\
\hline 1 & & & 4,678787879 & 1 \\
\hline 2 & & & 4,678787879 & 5 \\
\hline 3 & $30 \mathrm{mca}$ & $4 \mathrm{hrs}$ & 4,703030303 & 15 \\
\hline 4 & & & 4,896969697 & 30 \\
\hline
\end{tabular}


Cuadro 2: Radio de humedecimiento y volumen en Suelos Francos.

\begin{tabular}{|c|c|c|c|c|}
\hline \multicolumn{5}{|c|}{$\cdot$} \\
\hline Emisor & Presión & Tiempo & Volumen (L) & Radio (cm) \\
\hline 1 & & & 4,48676768 & 1 \\
\hline 2 & & & 4,36545455 & 4 \\
\hline 3 & $30 \mathrm{mca}$ & $4 \mathrm{hrs}$ & 4,68252526 & 12 \\
\hline 4 & & & 4,86282829 & 25 \\
\hline 5 & & & 5,12080809 & 38 \\
& & & & \\
\hline
\end{tabular}

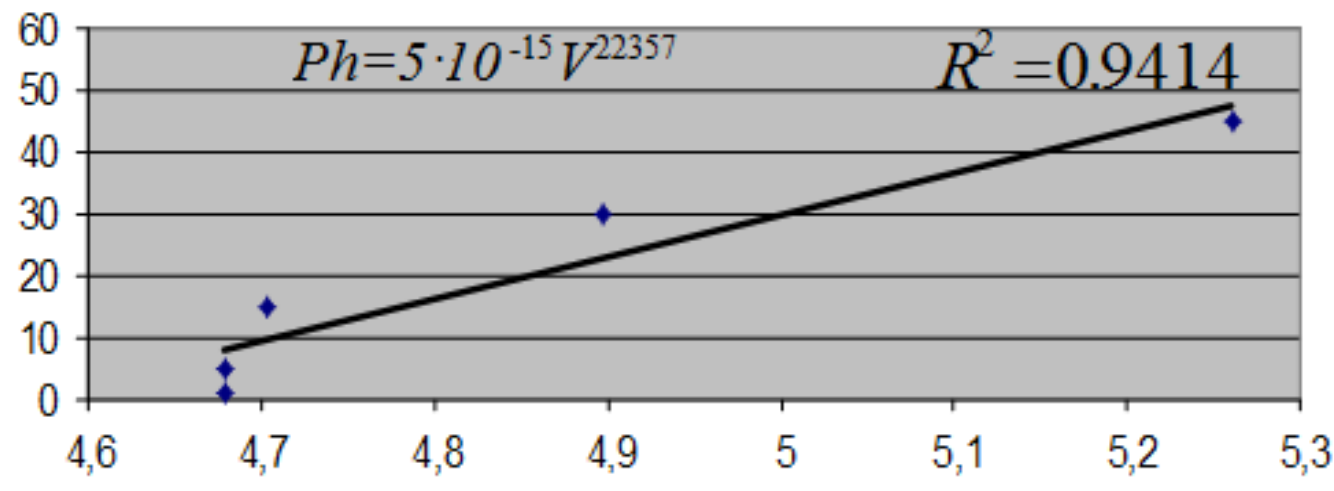

Figura 2. Caudal de descarga y radio de humedecimiento en Suelos Francos 


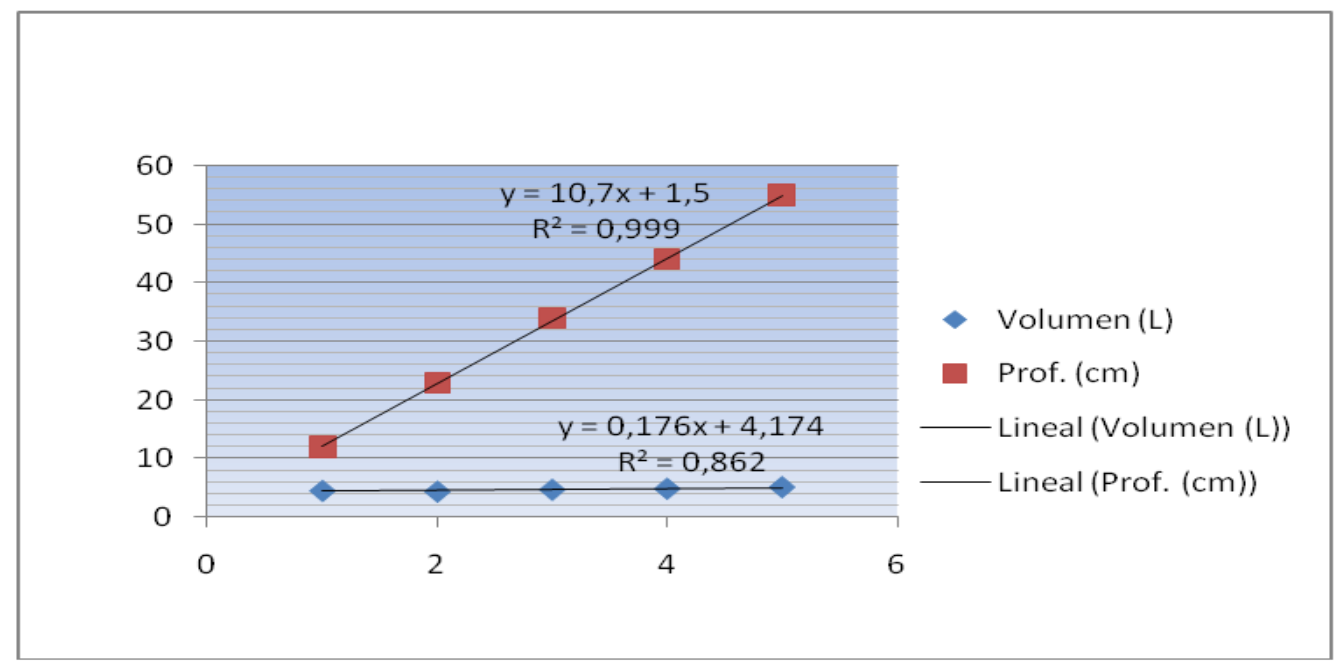

Figura 3. Profundidad de humedecimiento y volumen de agua aplicado

En los cuadros 3 y 4, y en la figuras 4 y 5 se presentan los resultados obtenidos de la relación entre la profundidad de humedecimiento y el volumen de agua aplicada por el emisor DRIPNET® en suelos ferralíticos rojos eutricos y en los suelos Francos. La ecuación de regresión obtenida de mayor ajuste fue también de tipo exponencial y de la forma siguiente.

$$
P h=a \cdot V^{m}
$$

Dónde:

Ph. - Profundidad de humedecimiento $(\mathrm{cm})$

v. - Volumen de agua aplicada (L)

a y m. - Valores obtenidos al ajustar la ecuación de regresión

El coeficiente de correlación obtenido de la ecuación 3.2 alcanzó 96 \% lo cual indica la estrecha relación existente entre las variable consideradas; Es decir la profundidad de humedecimiento esta íntimamente relacionado con el volumen de agua aplicado, estos resultados son similares a los obtenidos por Capra et al, (2005) y Porto et al, (2006).

Cabe señalar que las relaciones presentadas, estiman manera confiable el volumen de agua necesario para humedecer de manera satisfactoria la zona radicular de la caña de azúcar, en cada uno de sus diferentes estadios de fenológicos lo que evidentemente permite disminuir de forma 
significativa las pérdidas por percolación profunda. Finalmente se pretende señalar que el conocimiento de las formas y dimensiones del bulbo húmedo obtenido en este trabajo a partir de un emisor, permitirá determinar con exactitud el número de emisores necesarios para humedecer un determinado volumen de suelo y lo cual evidentemente influirá de manera significativa en la inversión inicial para el establecimiento del sistema de microirrigación necesario para el cultivo de la caña de azúcar.

Cuadro 3: Datos de la profundidad de humedecimiento vs. Volumen de agua aplicada al suelo por el emisor en suelos Ferralíticos Rojos.

\begin{tabular}{ccccc}
\hline Emisor & Presión & Tiempo & Volumen (L) & Prof. (cm) \\
\hline 1 & & & 4,632046 & 10 \\
\hline 2 & & & 4,632046 & 20 \\
\hline 3 & $30 \mathrm{mca}$ & 4 hrs. & 4,656047 & 30 \\
\hline 4 & & & 4,848048 & 40 \\
\hline 5 & & & 5,208052 & 50 \\
\hline
\end{tabular}

Cuadro 4: Datos de la profundidad de humedecimiento vs. Volumen de agua aplicada al suelo por el emisor en suelos Francos.

\begin{tabular}{ccccc}
\hline Emisor & Presión & Tiempo & Volumen $(\mathbf{L})$ & Prof. $(\mathbf{c m})$ \\
\hline 1 & & & 4,48676768 & 12 \\
\hline 2 & & & 4,36545455 & 23 \\
\hline 3 & $30 \mathrm{mca}$ & 4 hrs. & 4,68252526 & 34 \\
\hline 4 & & & 4,86282829 & 44 \\
\hline 5 & & & 5,12080809 & 55 \\
\hline
\end{tabular}




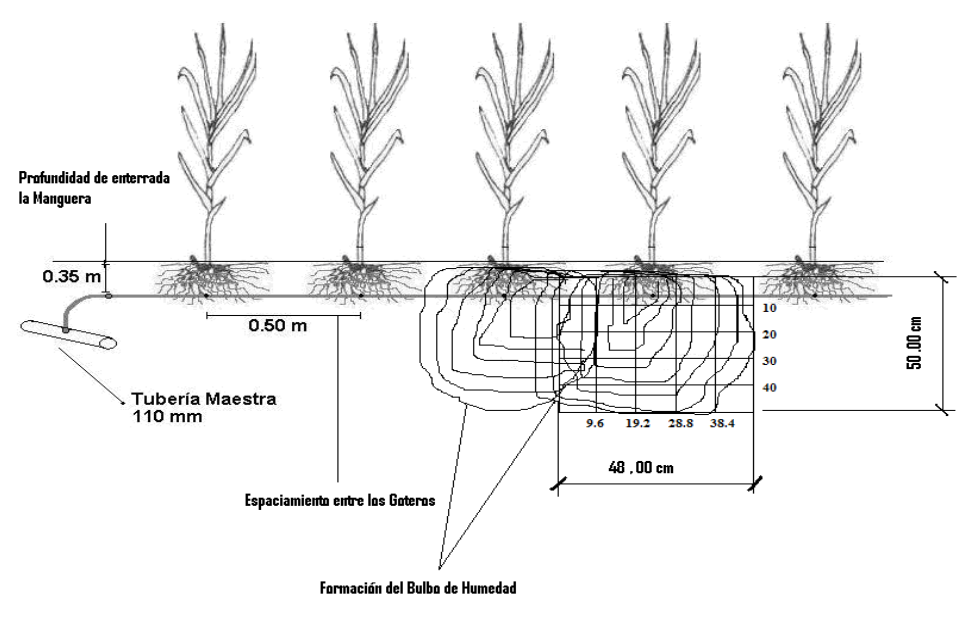

Figura 4. Gráfico de Comportamiento del cono de humedecimiento en suelos ferralíticos rojos.

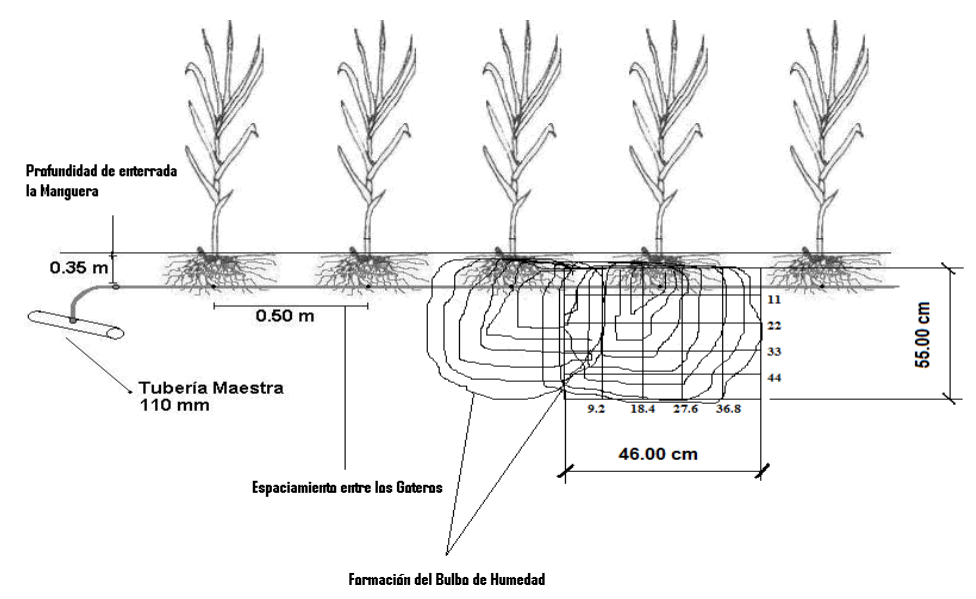

Figura 5. Gráficos de Comportamiento del Cono de Humedecimiento para suelos Francos.

\section{CONCLUSIONES}

Los resultados obtenidos demuestran que se logra una adecuada relación entre el caudal suministrado por el emisor y el Humedecimiento tanto vertical como Horizontal del Bulbo de Humedad a través de los emisores DRIPNET®. Las ecuaciones de regresión obtenida fueron relacionadas acertadamente del tipo exponencial bajo diferentes presiones ejercidas al sistema al igual que las ecuaciones Lineales obtenidas, tanto para los suelos Ferralíticos Rojos como los suelos Francos en diferentes intervalos de tiempo además de los valores obtenidos en cuanto a volumen aplicado, profundidad de humedecimiento y radio de humedecimiento. 


\section{REFERENCIAS}

Aidi, O. (2007). Irrifrance. Catalogo.

Bliesser y Keller et al (2000) y (2001). Clasificación Hidráulica de los Goteros.

Medina J. (1997). Riego por Goteo. $4^{a}$ ed. Madrid, España. Ediciones Mundi-Prensa; 302p.

Mujica A. y R. Veliz (2003). Criterio de diseño para minimizar el costo de tuberías dotadas de salidas múltiples. VI Congreso Internacional de Ingeniería Hidráulica. Ciego de Ávila.

Porto et al. (2006). Relación obtenida entre el bulbo de humedecimiento y el volumen aplicado utilizando la tecnología del riego por goteo.

Tornería M. (2005). Estudio del comportamiento del riego localizado subterráneo, en comparación con el riego localizado superficial Disponible en: http://www.Monografías.com/riegosubterr/riego-subterr.shtml. [Consultado el 24 de noviembre del 2014]. 